\title{
Kaluza Ansatz applied to Eddington inspired Born-Infeld Gravity
}

\author{
Karan Fernandes* and Amitabha Lahirit \\ S. N. Bose National Centre for Basic Sciences, \\ Block-JD, Sector III, Salt Lake, Kolkata-700098, INDIA.
}

(Dated: September 3, 2018)

\begin{abstract}
We apply Kaluza's procedure to Eddington-inspired Born-Infeld action in gravity in five dimensions. The resulting action contains, in addition to the usual four-dimensional actions for gravity and electromagnetism, nonlinear couplings between the electromagnetic field strength and curvature. Considering the spherically symmetric solution as an example we find the lowest order corrections for the Reissner-Nordström metric and the electromagnetic field.
\end{abstract}

*Electronic address: karan12t@bose.res.in

${ }^{\dagger}$ Electronic address: amitabha@bose.res.in 


\section{INTRODUCTION}

Eddington-Born-Infeld gravity arose out of a desire to find a gravitational analog of the determinantal action for electromagnetism proposed by Born and Infeld [1], with the hope that such an action would tame the singularities arising in gravity in much the same way as the Born-Infeld action does for electromagnetism. Early approaches in this area [2 4] ] proposed a determinantal Lagrangian, of the same form as the Born-Infeld Lagrangian, but with the electromagnetic tensor being replaced by the curvature tensor. In particular, models with the general structure suggested in [3] has been investigated over the years for its cosmological implications [5], has been shown to indeed alleviate the initial cosmological singularity that arises in standard General Relativity [5- 7], and has been shown to allow the regulation of the Schwarzschild singularity for positive energies [8]. In taking this theory to be not purely metric, but rather metric-affine [6], it has been suggested that it has novel implications in the matter coupling paradigm [9, 10]. However, as has been demonstrated [11], if the theory is taken to be metric-affine, it still leads to an effective metric theory upon further expansion. As such, we do not believe that the problem of coupling matter to gravity in this theory has been resolved or even adequately addressed. It is to address this issue that we have undertaken the present work.

We will work with Eddington-inspired Born-Infeld theory, with an action similar to that of [6], but in five dimensions. This is then reduced to four dimensions à la Kaluza by compactifying one dimension on a circle. We find corrections to the four-dimensional EddingtonBorn-Infeld theory, highly nonlinear terms which can be written in the form of infinite sums.

In Sec. II we provide a brief review of the Eddington-Born-Infeld Lagrangian and its equations of motion. We compare the purely affine Eddington action and the metric-affine action of Born and Infeld, written in the form of [6]. In the metric-affine theory, the equation of motion allows the affinity to be written as a function of the metric, so finally we have an equation for the metric only. It turns out that the equations of motion obtained from the two theories are equivalent, at least in regions of low curvature. In Sec. III, we go over the Kaluza procedure and use it to reduce a five-dimensional Eddington-inspired Born-Infeld theory to four dimensions. In Sec. IV], we derive the four-dimensional equations of motion due to this action. We find deviations from the gravitational equations as well as from the equations of motion for the electromagnetic field $A_{\mu}$ compared to the case when the 
electromagnetic field action is simply added to the gravitational action.

\section{THE EDDINGTON BORN-INFELD ACTION}

Faced with the problem of quantizing the electromagnetic field, while at the same time ensuring that the theory remain non-singular at short distances, Born and Infeld [1] introduced the action

$$
S_{B I}=\int d^{4} x b^{2}\left[\sqrt{-\operatorname{det}\left(g_{\mu \nu}\right)}-\sqrt{-\operatorname{det}\left(g_{\mu \nu}+b^{-1} F_{\mu \nu}\right)}\right],
$$

where $g_{\mu \nu}$ is the metric tensor in a flat spacetime, $F_{\mu \nu}$ is the electromagnetic field strength tensor, and $b$ is a constant which ensures that higher order terms of $F_{\mu \nu}$ get smoothed out in the expansion of the square root of the determinant. This theory, while being a nonlinear generalization of Maxwell's, has a number of promising features which ensures its viability. In particular these include the absence of birefringence in wave propagation and duality invariance $[12$ 16].

Since any attempt to quantize gravity faces an insurmountable problem with divergences, it is tempting to try the Born-Infeld route of ameliorating classical short-distance singularities. A determinantal action for gravity had been earlier proposed by Eddington [17],

$$
S_{E d d}=\int d^{4} x \sqrt{\left|\operatorname{det}\left(R(\Gamma)_{\mu \nu}\right)\right|} .
$$

Here $R(\Gamma)_{\mu \nu}$ is the symmetric part of the Ricci tensor constructed as a function of a torsionless affine connection $\Gamma_{\beta \gamma}^{\alpha}$, but in this action the $\Gamma^{\prime}$ 's are treated as independent fields, and not as functions of the metric and its derivatives. Since Eq. (2.2) is purely affine, we will denote $R(\Gamma)_{\mu \nu}$ as simply $R_{\mu \nu}$. The Ricci tensor is in general non-symmetric, so we have to specify that we take its symmetric part, with inverse defined via

$$
R^{\nu \mu} R_{\mu \alpha}:=\delta_{\alpha}^{\nu}
$$

Varying $\Gamma$, we find the equation of motion,

$$
\nabla_{\alpha}\left(\sqrt{|\mathbf{R}|} R^{\mu \nu}\right)-\nabla_{\beta}\left(\sqrt{|\mathbf{R}|} R^{\beta(\mu} \delta_{\alpha}^{\nu)}\right)=0
$$

Here and in what follows, we have used boldfaced letters to indicate matrices, and $|\mathbf{A}|$ to mean the absolute value of the determinant of the matrix $\mathbf{A}$. We will adopt the matrix notation wherever convenient and when no confusion can arise, as in Eq. (2.4) above, where 
we have denoted the matrix of $R_{\mu \nu}$ by $\mathbf{R}$, and the determinant of the said matrix by $|\mathbf{R}|$. We will write $\mathbf{g}$ when we mean the matrix of $g_{\mu \nu}$, but det $\mathbf{g}$ will be written as $|g|$ in accordance with common practice. The second term in Eq. (2.4) vanishes identically, as can be seen by tracing over either $\alpha$ and $\mu$, or $\alpha$ and $\nu$. Thus we are left with the following equation of motion for the connection,

$$
\nabla_{\alpha}\left(\sqrt{|\mathbf{R}|} R^{\mu \nu}\right)=0
$$

This equation shows that $\nabla_{\alpha}$ is the connection for the 'metric' $R_{\mu \nu}$, and we may define the metric by a rescaling

$$
R_{\mu \nu}=\lambda g_{\mu \nu}
$$

Thus the action of Eq. (2.2) has Einstein spaces as its extremal points. The equation of motion is the same as what we get from the more familiar Einstein-Hilbert action with a cosmological constant in vacuum,

$$
S_{E H}=\frac{1}{16 \pi} \int d^{4} x \sqrt{|g|}(R-2 \Lambda)
$$

provided we set $\lambda=\Lambda$. Here and below, we choose units in which $G=1$.

Eddington's theory thus reproduces Einstein's equation with a cosmological constant, but only in the absence of matter. One way of including matter is to generalize the action in the manner of Deser and Gibbons [3],

$$
S_{D G}=\int d^{4} x \sqrt{\left|\operatorname{det}\left(g_{\mu \nu}+R_{\mu \nu}+X_{\mu \nu}\right)\right|}
$$

$X_{\mu \nu}$ contains terms quadratic or higher in the curvature, a 'fudge tensor' introduced by hand in order to cancel out the quadratic curvature terms that arise out of expanding the determinant, and hence to render the theory ghost free. Matter can then be added to the theory via a contribution to $X_{\mu \nu}$ from the matter fields, e.g. $b F_{\mu \nu}$ for the Maxwell field.

A different approach was taken by Banados and Ferreira [6], who introduced, based on earlier investigations [19 21], what is now known as the Eddington-inspired Born-Infeld action,

$$
S_{E i B I}=\frac{1}{8 \pi \kappa} \int d^{4} x[\sqrt{|\mathbf{g}+\kappa \mathbf{R}(\Gamma)|}-\lambda \sqrt{|g|}],
$$

where $\kappa>0$ is a dimensionful constant. Here again $R_{\mu \nu}$ is a function of an independent connection $\Gamma_{\mu \nu}^{\alpha}$, and thus now there are two equations of motion - one from varying with 
respect to $g_{\mu \nu}$, and one from varying with respect to the connection $\Gamma_{\mu \nu}^{\alpha}$. Let us consider the case where the matter action is added as a separate term,

$$
S=S_{E i B I}\left[g_{\mu \nu}, \Gamma\right]+S_{M}\left[g_{\mu \nu}, \Gamma, \Psi\right]
$$

In the general formalism, the matter action $S_{M}$ need not be standard or minimal, but can also depend on the independent connection $\Gamma$. When $\kappa R_{\mu \nu} \gg g_{\mu \nu}$, the action of Eq. (2.9) becomes proportional to Eq. (2.2), and we get Einstein's equations with cosmological constant $\Lambda$ by setting $\kappa=\frac{16 \pi}{\Lambda}$. In finding this limit, we simply consider $\frac{\lambda}{\kappa} \sqrt{|g|}$ to be negligible in comparison with $\kappa \sqrt{|\mathbf{R}|}$.

On the other hand, when $\kappa R_{\mu \nu} \ll g_{\mu \nu}$, we can expand the determinant in a power series. Then $\kappa$ counts the power of curvature appearing in each term of the expansion. At the lowest order we find the Einstein-Hilbert action Eq. (2.7), as we should, provided we set $\lambda=\kappa \Lambda+1$. Since in this paper we are concerned with corrections to Einstein gravity stemming from Eq. (2.9), we will fix $\lambda=\kappa \Lambda+1$ in what follows. To understand the difficulties of adding matter to this theory, let us follow [6] for the moment and take $S_{M}$ in Eq. (2.10) to be a standard matter action.

Then the stress energy tensor $T_{\mu \nu}$ is calculated by varying $S_{M}$ with respect to the metric,

$$
T_{\mu \nu}:=-\frac{2}{\sqrt{|g|}} \frac{\delta S_{M}}{\delta g^{\mu \nu}}
$$

For numerical factors, we will adopt the conventions of [22], where the electromagnetic stress energy tensor and the Maxwell action take the following form

$$
S_{E M}=-\frac{1}{16 \pi} \int d^{4} x \sqrt{|g|} F_{\alpha \beta} F^{\alpha \beta}, \quad T_{\mu \nu}=\frac{1}{4 \pi}\left(F_{\mu \alpha} F_{\nu}{ }^{\alpha}-\frac{1}{4} F_{\alpha \beta} F^{\alpha \beta} g_{\mu \nu}\right) .
$$

These will be relevant in the sections to follow. For now, we will continue the general exposition for any matter field, for which only Eq.(2.11) will be of relevance.

With the assumption of a standard matter action, we obtain the following equations of motion from Eq. (2.10), after varying with respect to the metric and the connection (both being independent of each other at this point)

$$
\begin{gathered}
\sqrt{|\mathbf{g}+\kappa \mathbf{R}(\Gamma)|}\left((\mathbf{g}+\kappa \mathbf{R}(\Gamma))^{-1}\right)^{\mu \nu}-(\kappa \Lambda+1) \sqrt{|g|} g^{\mu \nu}=-8 \pi \kappa \sqrt{|g|} T^{\mu \nu} \\
\nabla_{\alpha}\left(\sqrt{|\mathbf{g}+\kappa \mathbf{R}(\Gamma)|}\left((\mathbf{g}+\kappa \mathbf{R}(\Gamma))^{-1}\right)^{\mu \nu}\right)=0
\end{gathered}
$$

As explained earlier, the boldfaced letters symbolize the corresponding matrices. 
Since the matter action is independent of the connection $\Gamma$, it is possible to solve for the connection in the same way as was done in the Eddington case. We take $q_{\mu \nu}=g_{\mu \nu}+\kappa R(\Gamma)_{\mu \nu}$, and require that it satisfy Eq. (2.14). This gives a connection

$$
\Gamma_{\beta \gamma}^{\alpha}=\frac{1}{2} q^{\alpha \mu}\left[q_{\beta \mu, \gamma}+q_{\gamma \mu, \beta}-q_{\beta \gamma, \mu}\right]
$$

and Eq. (2.13) takes the form

$$
\sqrt{|\mathbf{q}|} q^{\mu \nu}=(\kappa \Lambda+1) \sqrt{|g|} g^{\mu \nu}-8 \pi \kappa \sqrt{|g|} T^{\mu \nu},
$$

where $q^{\mu \nu}=\left((\mathbf{g}+\kappa \mathbf{R}(\Gamma))^{-1}\right)^{\mu \nu}$. The left hand side of this equation depends on both the metric and the independent connection, since the auxilliary metric $q_{\mu \nu}$ introduced here is a function of both $g_{\mu \nu}$ and $R(\Gamma)_{\mu \nu}$, whereas the right hand side depends only on the metric. This suggests that the connection is not truly independent of the metric; and this is indeed the case, as was shown in [6, 11]. First we find the determinant of Eq. (2.16), for which we get the following expression

$$
|\mathbf{q}|=|g|^{2}\left(\left|(1+\kappa \Lambda) \mathbf{g}^{-1}-8 \pi \kappa \mathbf{g}^{-1} \mathbf{T g}^{-1}\right|\right)
$$

Substituting this in Eq. (2.16), we find the following expression for $q_{\mu \nu}$,

$$
\mathbf{q}=\sqrt{|g|} \sqrt{\left|(1+\kappa \Lambda) \mathbf{g}^{-1}-8 \pi \kappa \mathbf{g}^{-1} \mathbf{T g}^{-1}\right|}\left((\kappa \Lambda+1) \mathbf{g}^{-1}-8 \pi \kappa \mathbf{g}^{-1} \mathbf{T g}^{-1}\right)^{-1}
$$

We can now expand this result by using the standard formulæ for the square root of the determinant, $(|\mathbf{I}+\mathbf{A}|)^{\frac{1}{2}}=1+\frac{\operatorname{tr}(\mathbf{A})}{2}+\frac{(\operatorname{tr}(\mathbf{A}))^{2}}{8}-\frac{\operatorname{tr}\left(\mathbf{A}^{2}\right)}{4}+\mathcal{O}\left(\mathbf{A}^{3}\right)$, and the inverse of a sum of matrices, $(\mathbf{A}+\mathbf{B})^{\mu \nu}=A^{\mu \nu}-A^{\mu \alpha} B_{\alpha \beta} A^{\beta \nu}+A^{\mu \alpha} B_{\alpha \beta} A^{\beta \gamma} B_{\gamma \delta} A^{\delta \nu}+\mathcal{O}\left(\mathbf{B}^{3}\right)$, where $\mathbf{I}$ is the identity matrix. Using these, we acquire the expression for $R(\Gamma)_{\mu \nu}$ as

$$
R(\Gamma)_{\mu \nu}=\Lambda g_{\mu \nu}+8 \pi\left[T_{\mu \nu}-\frac{1}{2} T g_{\mu \nu}\right]+64 \pi^{2} \kappa\left[S_{\mu \nu}-\frac{1}{4} S g_{\mu \nu}\right]+\mathcal{O}\left(\kappa^{2}\right)
$$

where $S_{\mu \nu}$ is given by

$$
S_{\mu \nu}=T_{\mu \alpha} T_{\nu}^{\alpha}-\frac{1}{2} T T_{\mu \nu}
$$

However, Eq. (2.19) can also be used to find the expression for the Ricci tensor as a function of the metric. Inverting Eq. (2.18) gives us the expression for $q^{\mu \nu}$. Keeping only terms to order $\kappa$, we find

$$
q^{\mu \nu}=g^{\mu \nu}-\kappa \tau^{\mu \nu}+\mathcal{O}\left(\kappa^{2}\right)
$$


where $\tau_{\mu \nu}=\Lambda g_{\mu \nu}+8 \pi\left[T_{\mu \nu}-\frac{1}{2} T g_{\mu \nu}\right]$. One can now use the expressions for $q_{\mu \nu}$ and $q^{\mu \nu}$ in Eq. (2.15), and expand up to order $\kappa$. After a bit of algebra, this produces the expression

$$
\Gamma_{\beta \gamma}^{\alpha}=\left\{\begin{array}{c}
\alpha \\
\beta \gamma
\end{array}\right\}+\frac{1}{2} \kappa q^{\alpha \delta}\left(R(\Gamma)_{\delta \beta ; \gamma}+R(\Gamma)_{\gamma \delta ; \beta}-R(\Gamma)_{\beta \gamma ; \delta}\right)
$$

where the semicolon in the subscript implies a covariant derivative calculated using $\left\{\begin{array}{c}\alpha \\ \beta \gamma\end{array}\right\}$, the Christoffel symbols corresponding to $g_{\mu \nu}$. The Ricci tensor calculated using these $\Gamma$ is given by

$$
R(\Gamma)_{\mu \nu}=R(g)_{\mu \nu}+\frac{1}{2} \kappa g^{\alpha \beta}\left(R(\Gamma)_{\alpha \mu ; \nu \beta}+R(\Gamma)_{\alpha \nu ; \mu \beta}-R(\Gamma)_{\mu \nu ; \alpha \beta}-R(\Gamma)_{\alpha \beta ; \mu \nu}\right)+\mathcal{O}\left(\kappa^{2}\right) .
$$

Equating the right hand sides of Eq. (2.19) and Eq. (2.23) we find

$$
\begin{aligned}
R(g)_{\mu \nu}=\Lambda g_{\mu \nu}+ & 8 \pi\left[T_{\mu \nu}-\frac{1}{2} T g_{\mu \nu}\right]+64 \pi^{2} \kappa\left[S_{\mu \nu}-\frac{1}{4} S g_{\mu \nu}\right] \\
& +\frac{1}{2} \kappa\left[\nabla_{\mu} \nabla_{\nu} \tau-2 \nabla^{\alpha} \nabla_{(\mu} \tau_{\nu) \alpha}+\square \tau_{\mu \nu}\right]+\mathcal{O}\left(\kappa^{2}\right)
\end{aligned}
$$

where we have written $\square \equiv \nabla_{\mu} \nabla^{\mu}$, and $\tau_{\mu \nu}$ is as defined above. We see that this expression contains at least third derivatives of the matter fields. A consequence of this is that there could exist singularities in the curvature invariants should the matter distribution be discontinuous enough, and that there are surface singularities, in the case of polytropic stars [11]. This has brought the viability of this theory into question.

We note here that there is another way of writing the equation of motion, which follows from the fact that Eq. (2.23) implies that to leading order, $R(\Gamma)_{\mu \nu}=R(g)_{\mu \nu}$, and all corrections to this are $\mathcal{O}(\kappa)$ or higher. Thus we can rewrite Eq. (2.23) as

$$
R(\Gamma)_{\mu \nu}=R(g)_{\mu \nu}+\frac{1}{2} \kappa g^{\alpha \beta}\left(R(g)_{\alpha \mu ; \nu \beta}+R(g)_{\alpha \nu ; \mu \beta}-R(g)_{\mu \nu ; \alpha \beta}-R(g)_{\alpha \beta ; \mu \nu}\right)+\mathcal{O}\left(\kappa^{2}\right) .
$$

Putting this back into Eq. (2.19), we can write the equation of motion as

$$
\begin{aligned}
R(g)_{\mu \nu}=\Lambda & g_{\mu \nu}+8 \pi\left[T_{\mu \nu}-\frac{1}{2} T g_{\mu \nu}\right]+64 \pi^{2} \kappa\left[S_{\mu \nu}-\frac{1}{4} S g_{\mu \nu}\right] \\
& -\frac{1}{2} \kappa g^{\alpha \beta}\left(R(g)_{\alpha \mu ; \nu \beta}+R(g)_{\alpha \nu ; \mu \beta}-R(g)_{\mu \nu ; \alpha \beta}-R(g)_{\alpha \beta ; \mu \nu}\right)+\mathcal{O}\left(\kappa^{2}\right)
\end{aligned}
$$

Any solution of Eq. (2.24) is a solution of Eq. (2.26) and vice versa. Although both these equations have been derived from Eq. (2.19) by neglecting $\mathcal{O}\left(\kappa^{2}\right)$ terms, it is obvious that we can follow the procedure to get two equations at higher order in $\kappa$ as well. While the $\kappa$ expansion in the first equation corresponds to a series in the stress-energy tensor and its derivatives, whereas the expansion in the second equation is one in curvature. 
Since Eq. (2.26) is written fully in terms of the Levi-Civita connection, we can use the relation between this connection and $R(g)_{\mu \nu}$ to rewrite it as

$$
\begin{aligned}
R_{\mu \nu}=\Lambda g_{\mu \nu}+8 & \pi\left(T_{\mu \nu}-\frac{1}{2} T g_{\mu \nu}\right)+64 \pi^{2} \kappa\left(S_{\mu \nu}-\frac{1}{4} S g_{\mu \nu}\right) \\
& +\frac{1}{2} \kappa\left[2 R_{\alpha \mu \beta \nu} R^{\alpha \beta}-2 R_{\mu \beta} R_{\nu}^{\beta}+\square R_{\mu \nu}\right]+\mathcal{O}\left(\kappa^{2}\right) .
\end{aligned}
$$

We have seen above that if we start from the metric-affine theory, the equations of motion naturally lead to a purely metric expression for the usual Ricci tensor. It is thus natural to investigate what the equations of motion might be if we started with a purely metric version of the theory. The action is the same as in Eq. (2.10), but with $R_{\mu \nu}=R(g)_{\mu \nu}$,

$$
S=\frac{1}{8 \pi \kappa} \int d^{4} x[\sqrt{|\mathbf{g}+\kappa \mathbf{R}(g)|}-\lambda \sqrt{|g|}]+S_{M}\left[g_{\mu \nu}, \Psi\right] .
$$

As before, we set $\lambda=\kappa \Lambda+1$, and vary this action with respect to $g_{\mu \nu}$, to find

$$
\begin{aligned}
\delta S=\frac{1}{8 \pi \kappa} \int d^{4} x\left[\frac{1}{2} \sqrt{|\mathbf{g}+\kappa \mathbf{R}|}(\mathbf{g}+\kappa \mathbf{R})^{-1}\right)^{\mu \nu}\left(\delta g_{\mu \nu}+\kappa \delta R_{\mu \nu}\right) \\
\left.-\frac{1}{2}(\kappa \Lambda+1) \sqrt{|g|} g^{\mu \nu} \delta g_{\mu \nu}+\frac{8 \pi \kappa}{2} \sqrt{|g|} T^{\mu \nu} \delta g_{\mu \nu}\right] \\
=\frac{1}{16 \pi \kappa} \int d^{4} x\left[\sqrt{|\overline{\mathbf{q}}|} \bar{q}^{\mu \nu}+\kappa H^{\mu \nu}-(\kappa \Lambda+1) \sqrt{|g|} g^{\mu \nu}+8 \pi \kappa \sqrt{|g|} T^{\mu \nu}\right] \delta g_{\mu \nu},
\end{aligned}
$$

where we have defined $\bar{q}_{\mu \nu}=g_{\mu \nu}+\kappa R_{\mu \nu}$, to distinguish it from the earlier case where we had $R(\Gamma)_{\mu \nu}$. We have also defined $H^{\mu \nu}$, which in terms of the notation just introduced, is given by

$$
H^{\mu \nu}=\frac{1}{2}\left[\nabla_{\alpha} \nabla^{\mu}\left(\sqrt{|\overline{\mathbf{q}}|} \bar{q}^{\alpha \nu}\right)+\nabla_{\alpha} \nabla^{\nu}\left(\sqrt{|\overline{\mathbf{q}}|} \bar{q}^{\mu \alpha}\right)-\nabla_{\alpha} \nabla_{\beta}\left(\sqrt{|\overline{\mathbf{q}}|} \bar{q}^{\beta \alpha} g^{\mu \nu}\right)-\square\left(\sqrt{|\overline{\mathbf{q}}|} \bar{q}^{\mu \nu}\right)\right] .
$$

In going from the first line to the second line of Eq. (2.29), we made use of the Palatini identity $\delta R_{\mu \nu}=\nabla_{\alpha}\left(\delta \Gamma_{\mu \nu}^{\alpha}\right)-\nabla_{\mu}\left(\delta \Gamma_{\alpha \nu}^{\alpha}\right)$, and exploited the Leibniz rule for covariant derivatives to eliminate total derivatives.

We will now make use of the equation of motion that comes out of this,

$$
\sqrt{|\overline{\mathbf{q}}|} \bar{q}^{\mu \nu}+\kappa H^{\mu \nu}-(\kappa \Lambda+1) \sqrt{|g|} g^{\mu \nu}=-8 \pi \kappa \sqrt{|g|} T^{\mu \nu}
$$

to find an expression for $R_{\mu \nu}$. We can substitute $\bar{q}_{\mu \nu}$ in the expression for $H^{\mu \nu}$ above to find

$$
\begin{aligned}
H^{\mu \nu} & =-\frac{1}{2} \kappa \sqrt{|g|}\left[2 R_{\alpha \delta}^{\mu \nu} R^{\alpha \delta}+2 R_{\delta}^{\mu} R^{\nu \delta}-\square R^{\mu \nu}+\frac{1}{2} g^{\mu \nu} \square R\right]+\mathcal{O}\left(\kappa^{2}\right) \\
& \equiv \sqrt{|g|} \tilde{H}^{\mu \nu} .
\end{aligned}
$$


Eq. (2.32) clearly shows that there are no $\mathcal{O}\left(\kappa^{0}\right)$ terms in $H^{\mu \nu}$ in the lowest order. Eq. (2.31) will thus also yield Einstein's equation with a cosmological constant in the case of $\kappa=0$. To make things more explicit, we proceed as before and acquire the determinant of Eq. (2.31)

$$
\overline{\mathbf{q}}=\sqrt{|g|} \sqrt{\left|(1+\kappa \Lambda) \mathbf{g}^{-1}-\kappa \mathbf{g}^{-1}(\tilde{\mathbf{H}}+8 \pi \mathbf{T}) \mathbf{g}^{-1}\right|}\left((\kappa \Lambda+1) \mathbf{g}^{-1}-\kappa \mathbf{g}^{-1}(\tilde{\mathbf{H}}+8 \pi \mathbf{T}) \mathbf{g}^{-1}\right)^{-1} .
$$

The expansion of Eq.(2.33) up to order $\kappa$ reveals the following expression

$$
\begin{aligned}
R_{\mu \nu}=\Lambda g_{\mu \nu}+8 & \pi\left(T_{\mu \nu}-\frac{1}{2} T g_{\mu \nu}\right)+64 \pi^{2} \kappa\left(S_{\mu \nu}-\frac{1}{4} S g_{\mu \nu}\right) \\
& +\frac{1}{2} \kappa\left[2 R_{\alpha \mu \beta \nu} R^{\alpha \beta}-2 R_{\mu \beta} R_{\nu}^{\beta}+\square R_{\mu \nu}\right]+\mathcal{O}\left(\kappa^{2}\right) .
\end{aligned}
$$

This is the same equation that we found in the metric-affine theory when we wrote the equation of motion in terms of quantities derived from $g_{\mu \nu}$. Thus the metric theory and the metric-affine theory are equivalent.

This observation brings us to the main motivation for this work. We ask if there is a natural way of incorporating the matter part of the action into the theory other than simply adding it, such that we still reproduce Einstein's theory in the weak limit. There exist still further proposals over the incorporation of matter in this theory. In [20, 21], $R(\Gamma)_{\mu \nu}$ was allowed to have an antisymmetric component, leading to the action for a massive vector field. In a different approach [9, 10], matter was coupled to the "metric" $q_{\mu \nu}$ in the field equations Eq. (2.13) and Eq. (2.14). Since the vacuum equations are the same as in usual general relativity, this coupling plays out only in signficantly matter dense regions, as in the interior of stars.

Here we take a geometric approach, while staying close to the original Born-Infeld idea of ameliorating singularities. Our approach will be to use Kaluza's idea [23] of unifying gravity and electromagnetism in a five-dimensional theory of gravitation, and apply it to the five-dimensional Eddington-Born-Infeld theory. Since this procedure deals only with the five-dimensional metric, we will necessarily deal with the metric version of the EddingtonBorn-Infeld action. However, as we have seen in this section, the two approaches agree at least to $\mathcal{O}(\kappa)$, so we may consider the resulting action a natural way of incorporating electromagnetic fields in the four-dimensional Eddington-inspired Born-Infeld gravitational theory. 


\section{THE KALUZA ANSATZ}

We start by writing the metric in the form

$$
\hat{g}_{A B}=\left(\begin{array}{cc}
g_{\mu \nu}+\alpha^{2} \Phi^{2} A_{\mu} A_{\nu} & \alpha \Phi^{2} A_{\mu} \\
\alpha \Phi^{2} A_{\nu} & \Phi^{2}
\end{array}\right) .
$$

Here and later, uppercase Latin indices are five-dimensional, $A, B, \cdots=0, \cdots, 3,5$, while Greek indices are four-dimensional, $\mu, \nu, \cdots=0, \cdots, 3$. Five-dimensional objects will be written with hats, and $\alpha$ is a parameter which will be fixed later. The inverse of this matrix is

$$
\hat{g}^{A B}=\left(\begin{array}{cc}
g^{\mu \nu} & -\alpha A^{\mu} \\
-\alpha A^{\nu} & \alpha^{2} A^{\gamma} A_{\gamma}+\frac{1}{\Phi^{2}}
\end{array}\right) .
$$

While the consequences of the including the scale of the fifth dimension as an independent scalar field [24] is interesting in its own right, our interest lies in the coupling of electromagnetism to gravity, so we will set $\Phi=1$. In Appendix $\mathrm{A}$ we have given the expression for the Ricci scalar for a non-trivial $\Phi$. We will construct the Eddington-Born-Infeld action for the five-dimensional metric theory, i.e. we will write Eq. (2.29) for the above metric ansatz and derive some of its consequences.

The Ricci tensor components are calculated in a straightforward manner,

$$
\begin{aligned}
& \hat{R}_{\mu \nu}=R_{\mu \nu}+\frac{1}{4} \alpha^{4} F^{\beta \gamma} F_{\beta \gamma} A_{\mu} A_{\nu}-\frac{1}{2} \alpha^{2}\left(A_{\mu} \nabla_{\beta} F_{\nu}^{\beta}+A_{\nu} \nabla_{\beta} F_{\mu}^{\beta}+F_{\beta \mu} F_{\nu}^{\beta}\right) \\
& \hat{R}_{\mu 5}=\frac{1}{4} \alpha^{3} F^{\beta \gamma} F_{\beta \gamma} A_{\mu}-\frac{1}{2} \alpha\left(\nabla_{\beta} F_{\mu}^{\beta}\right), \quad \hat{R}_{55}=\frac{1}{4} \alpha^{2} F^{\beta \gamma} F_{\beta \gamma},
\end{aligned}
$$

giving the Ricci scalar,

$$
\hat{R}=R-\frac{\alpha^{2}}{4} F^{\beta \gamma} F_{\beta \gamma}
$$

If we write the radius of compactification of the fifth dimension as $\tilde{R}$, and the fivedimensional Newton's constant as $\hat{G}_{5}$, we find that setting

$$
\frac{2 \pi \tilde{R}}{\hat{G}_{5}}=\frac{1}{G}=1, \quad \alpha^{2}=4 G=4
$$

leads to the reduction of the five-dimensional Einstein-Hilbert action as

$$
\frac{1}{16 \pi \hat{G}_{5}} \int d^{5} x \sqrt{|\hat{g}|} \hat{R}=\frac{1}{16 \pi} \int d^{4} x \sqrt{|g|} R-\frac{1}{16 \pi} \int d^{4} x \sqrt{|g|} F^{\beta \gamma} F_{\beta \gamma} .
$$

The factor in front of the electromagnetic action agrees with our conventions, shown in Eq. (2.12). We will set this value of $\alpha^{2}$ in the remainder of the paper. 
We now write the Eddington-inspired Born-Infeld action in the metric form, i.e. the action of Eq. (2.28) without $S_{M}$, but in five dimensions. Then we will need to find two determinants - those of $\hat{g}_{A B}$ and $\hat{q}_{A B}=\hat{g}_{A B}+\kappa \hat{R}_{A B}$. Using the usual decomposition of a block matrix,

$$
\begin{aligned}
\operatorname{det}\left(\begin{array}{ll}
A & B \\
C & D
\end{array}\right) & =\operatorname{det}\left[\left(\begin{array}{cc}
A-B D^{-1} C & B D^{-1} \\
0 & 1
\end{array}\right)\left(\begin{array}{ll}
1 & 0 \\
C & 1
\end{array}\right)\left(\begin{array}{ll}
1 & 0 \\
0 & D
\end{array}\right)\right] \\
& =\operatorname{det}(D) \operatorname{det}\left(A-B D^{-1} C\right)
\end{aligned}
$$

we find that $\left|\operatorname{det}\left(\hat{g}_{A B}\right)\right|=\left|\operatorname{det}\left(g_{\mu \nu}\right)\right| \equiv|g|$. The other block matrix, $\hat{q}_{A B}$, has the following components

$$
\begin{aligned}
& \hat{q}_{\mu \nu}=g_{\mu \nu}+4 A_{\mu} A_{\nu}\left(1+\kappa F^{2}\right)+\kappa\left[R_{\mu \nu}-2\left(A_{\mu} \nabla_{\beta} F_{\nu}^{\beta}+A_{\nu} \nabla_{\beta} F_{\mu}^{\beta}+F_{\beta \mu} F_{\nu}^{\beta}\right)\right] \\
& \hat{q}_{5 \nu}=2 A_{\nu}\left(1+\kappa F^{2}\right)-\kappa \nabla_{\beta} F_{\nu}^{\beta}, \quad \hat{q}_{55}=1+\kappa F^{2},
\end{aligned}
$$

where we have written $F_{\mu \nu} F^{\mu \nu}=F^{2}$. Now we make a formal expansion in powers of $\kappa$ to find $\left(\hat{q}_{55}\right)^{-1}$, and get

$$
\left[q_{\mu \nu}-q_{\mu 5}\left(q_{55}\right)^{-1} q_{5 \nu}\right]=g_{\mu \nu}+\kappa\left(R_{\mu \nu}+2 F_{\mu \beta} F_{\nu}^{\beta}\right)+\nabla_{\delta} F_{\mu}^{\delta} \nabla_{\beta} F_{\nu}^{\beta} \sum_{n=0}^{\infty}(-1)^{n+1} \kappa^{n+2} F^{2 n} \text {. }
$$

Using this, we can write the Eddington-Born-Infeld action in the five-dimensional spacetime,

$$
\begin{aligned}
S & =\frac{1}{8 \pi \hat{G}_{5} \kappa} \int d^{5} x\left[\sqrt{\left|\left(\hat{g}_{A B}+\kappa \hat{R}_{A B}\right)\right|}-(\kappa \Lambda+1) \sqrt{|\hat{g}|}\right] \\
& =\frac{1}{8 \pi \kappa} \int d^{4} x\left[\sqrt{1+\kappa F^{2}} \times\right. \\
& \left.\sqrt{\left|g_{\mu \nu}+\kappa\left(R_{\mu \nu}+2 F_{\mu \beta} F_{\nu}^{\beta}\right)+\left(\nabla_{\delta} F_{\mu}^{\delta} \nabla_{\beta} F_{\nu}^{\beta}\right) \sum_{n=0}^{\infty}(-1)^{n+1} \kappa^{n+2} F^{2 n}\right|}-(\kappa \Lambda+1) \sqrt{g}\right] .
\end{aligned}
$$

We have used Eq. (3.5) in going from the first to the second line in Eq. (3.10), analogous to the Einstein-Hilbert treatment above. Remember that $\kappa$ counts the powers of curvature, so keeping terms up to some given order of $\kappa$ is the same as neglecting higher powers of curvature. We will be interested in determining the lowest order corrections to the equations of motion, which means that we need only expand to second order, i.e. $\mathcal{O}\left(\kappa^{2}\right)$. In order to 
get an $\mathcal{O}\left(\kappa^{2}\right)$ contribution from the first term, we need only consider the $n=0$ term in the sum. The action to this order is given by

$$
S=\frac{1}{8 \pi \kappa} \int d^{4} x\left[\sqrt{1+\kappa F^{2}} \sqrt{\left|g_{\mu \nu}+\kappa\left(R_{\mu \nu}+2 F_{\mu \beta} F_{\nu}^{\beta}\right)-\kappa^{2} \nabla_{\delta} F_{\mu}^{\delta} \nabla_{\beta} F_{\nu}^{\beta}\right|}-(\kappa \Lambda+1) \sqrt{|g|}\right] .
$$

\section{EQUATIONS OF MOTION}

We will first expand Eq. (3.11) up to first order in $\kappa$, before proceeding to its expansion to order $\kappa^{2}$. In expanding the determinant and varying the action, we also expect that linearity will ensure that we get the same equations of motion at the lowest order as Eq. (2.9), which is nothing but Einstein's equation with a cosmological constant.

If we expand all terms to first order $\kappa$, the action is given by

$$
S=\frac{1}{8 \pi \kappa} \int d^{4} x \sqrt{g}\left[\left(1+\frac{\kappa}{2} F^{2}\right)\left(1+\frac{\kappa}{2}\left(R-2 F^{2}\right)\right)-(\kappa \Lambda+1)+\mathcal{O}\left(\kappa^{2}\right)\right],
$$

from which we get the equations of motion

$$
G_{\mu \nu}=-\Lambda g_{\mu \nu}+8 \pi T_{\mu \nu}, \quad \nabla_{\alpha} F^{\alpha \nu}=0
$$

We thus recover the equations of motion of the Einstein-Maxwell theory, and dynamics is unaffected at the lowest order, as it should be.

Expanding to second order in $\kappa$ gives us

$$
\begin{gathered}
S=\frac{1}{8 \pi \kappa} \int d^{4} x \sqrt{g}\left[( 1 + \frac { \kappa F ^ { 2 } } { 2 } - \frac { \kappa ^ { 2 } F ^ { 4 } } { 8 } ) \left(1+\frac{\kappa}{2}\left(R-2 F^{2}\right)+\frac{\kappa^{2}}{8}\left(R^{2}+4 F^{4}-4 R F^{2}\right)\right.\right. \\
\left.-\frac{\kappa^{2}}{4}\left(R^{\alpha \beta} R_{\beta \alpha}+4 R^{\alpha \beta} F_{\beta \gamma} F_{\alpha}^{\gamma}+4 F^{\alpha \beta} F_{\beta \gamma} F^{\gamma \delta} F_{\delta \alpha}-2 \nabla_{\alpha} F^{\alpha \beta} \nabla^{\gamma} F_{\gamma \beta}\right)\right) \\
\left.-(\kappa \Lambda+1)+\mathcal{O}\left(\kappa^{3}\right)\right],
\end{gathered}
$$

which is extremized with respect to the inverse metric, to get the following equation of motion to order $\kappa$

$$
G_{\mu \nu}=-\Lambda g_{\mu \nu}+8 \pi T_{\mu \nu}+\kappa P_{\mu \nu}+\kappa Q_{\mu \nu}
$$

Here $G_{\mu \nu}$ is the usual Einstein tensor, $T_{\mu \nu}$ is the usual energy-momentum tensor of electro- 
dynamics, and

$$
\begin{aligned}
& P_{\mu \nu}=R_{\mu \alpha} R_{\nu}^{\alpha}-\frac{1}{2} R R_{\mu \nu}-\frac{1}{4} R_{\alpha \beta} R^{\alpha \beta} g_{\mu \nu}+\frac{1}{8} R^{2} g_{\mu \nu}+\frac{1}{2} \nabla_{\mu} \nabla_{\nu} R-\frac{1}{2} g_{\mu \nu} \square R \\
& \quad-\nabla_{\alpha} \nabla_{(\mu} R_{\nu)}^{\alpha}+\frac{1}{2} \square R_{\mu \nu}+\frac{1}{2} g_{\mu \nu} \nabla_{\alpha} \nabla_{\beta} R^{\alpha \beta} \\
& Q_{\mu \nu}=R F_{\mu \alpha} F_{\nu}^{\alpha}+\nabla_{\alpha} F_{\nu}^{\alpha} \nabla_{\beta} F_{\mu}^{\beta}+2 R_{\mu \alpha} F^{\alpha \beta} F_{\beta \nu}+2 F_{\mu \alpha} R^{\alpha \beta} F_{\beta \nu} \\
&+ 2 F_{\mu \alpha} F^{\alpha \beta} R_{\beta \nu}+8 F_{\mu \alpha} F^{\alpha \beta} F_{\beta \gamma} F_{\nu}^{\gamma}-2 \nabla_{(\mu}\left(F_{\nu) \beta} \nabla_{\alpha} F^{\alpha \beta}\right) \\
&-2 \nabla_{\alpha}\left(F_{(\mu}^{\alpha} \nabla_{|\beta|} F_{\nu)}^{\beta}\right)+F^{2} F_{\mu \beta} F_{\nu}^{\beta}+2\left(\nabla_{(\mu} F_{\nu) \beta}\right) \nabla_{\alpha} F^{\alpha \beta} \\
&-2 \nabla_{\alpha} \nabla_{(\mu}\left(F_{\nu) \beta} F^{\beta \alpha}\right)+\square\left(F_{\mu \beta} F_{\nu}^{\beta}\right)+g_{\mu \nu} \nabla_{\alpha} \nabla_{\beta}\left(F^{\alpha \gamma} F_{\gamma}^{\beta}\right) \\
&-\frac{1}{2} \nabla_{\mu} \nabla_{\nu} F^{2}+\frac{1}{2} g_{\mu \nu} \square F^{2}+\frac{1}{2} F^{2} R_{\mu \nu}-\frac{1}{4} g_{\mu \nu} R F^{2}-F_{\alpha \beta} F^{\beta \gamma} F_{\gamma \delta} F^{\delta \alpha} g_{\mu \nu} \\
&-F_{\alpha \beta} F^{\beta \gamma} R_{\gamma}^{\alpha} g_{\mu \nu}+\nabla_{\alpha}\left(F_{\beta}^{\alpha}{ }_{\beta} \nabla_{\gamma} F^{\gamma \beta}\right) g_{\mu \nu}-\frac{1}{8} F^{4} g_{\mu \nu}-\frac{1}{2}\left(\nabla_{\alpha} F_{\beta}^{\alpha}\right) \nabla_{\gamma} F^{\gamma \beta} g_{\mu \nu}
\end{aligned}
$$

Here $P_{\mu \nu}$ contain all the $\mathcal{O}(\kappa)$ terms which do not contain the field strength tensor, while $Q_{\mu \nu}$ terms are the $\mathcal{O}(\kappa)$ terms that do. Variation of the action with respect to $A_{\mu}$, gives the equation of motion

$$
\begin{gathered}
\nabla_{\alpha} F^{\alpha \nu}=-\kappa\left[\nabla_{\alpha}\left(F^{\alpha \nu}\left(\frac{1}{2} R+\frac{1}{2} F^{2}\right)\right)-4 \nabla_{\alpha}\left(F^{\alpha \beta} F_{\beta \gamma} F^{\gamma \nu}\right)-\nabla_{\alpha}\left(R^{\alpha \beta} F_{\beta}{ }^{\nu}-R^{\nu \beta} F_{\beta}{ }^{\alpha}\right)\right. \\
\left.-\frac{1}{2} \square\left(\nabla_{\alpha} F^{\alpha \nu}\right)+\frac{1}{2} \nabla_{\alpha} \nabla^{\nu}\left(\nabla_{\beta} F^{\beta \alpha}\right)\right]+\mathcal{O}\left(\kappa^{2}\right) .
\end{gathered}
$$

Even at first order in $\kappa$, what we get from the Eddington-Born-Infeld theory in five dimensions differs from what we would get by adding usual Maxwell action to the fourdimensional theory as in Eq. (2.9). This is true for all the equations derived thence, be it Eq. (2.19) derived via a Palatini variation, the expansion of that as in Eq. (2.24), or Eq. (2.34) derived through the variation of a completely metric theory. The difference lies primarily in the fact that we have couplings between the electromagnetic field strength and the curvature. Note however that if we set $F_{\mu \nu}=0$ in Eq. (4.4), the resulting equation agrees with Eq. (2.34).

Taking the trace of the equations brings out the difference rather dramatically. We have seen earlier that if we add electromagnetism as a separate matter action, the trace of Eq. (2.24) produces $R=4 \Lambda$, since the stress-energy tensor electromagnetism is traceless. The trace of Eq. (2.34) gives the same result if we formally expand $\left(1-\frac{1}{2} \kappa \square\right)^{-1}$. In contrast, 
the trace of Eq. (4.4) produces to order $\kappa$

$$
\begin{aligned}
R=4 \Lambda-\kappa\left[\frac{1}{2} F^{4}\right. & +\frac{1}{2} R F^{2}+2 R^{\alpha \beta} F_{\beta \gamma} F_{\alpha}^{\gamma}+4 F^{\alpha \beta} F_{\beta \gamma} F^{\gamma \delta} F_{\delta \alpha} \\
& \left.+\nabla_{\alpha} \nabla_{\beta}\left(R^{\alpha \beta}+2 F^{\alpha \delta} F_{\delta}{ }^{\beta}\right)-\square\left(R-\frac{1}{2} F^{2}\right)+\nabla_{\beta} F^{\beta \gamma} \nabla^{\alpha} F_{\alpha \gamma}\right] .
\end{aligned}
$$

Thus the Kaluza-Klein prescription leads to the incorporation of electromagnetic fields in the theory as expected, but also to novel non-trivial couplings which get naturally introduced because of the determinantal form of the action.

\section{ITERATIVE SOLUTIONS}

The equations of motion for the metric and the vector potential will have even more complicated couplings at higher orders of $\kappa$ as they come from a higher-order expansion of the action (3.10). However, it is possible to find solutions to these equations to any order in $\kappa$ via an iterative procedure, which we will now describe.

Let us rewrite the $\mathcal{O}(\kappa)$ equations of motion Eq. (4.4) and Eq. (4.7) as

$$
\begin{aligned}
\Lambda g_{\mu \nu} & =-G_{\mu \nu}+8 \pi T_{\mu \nu}-\kappa C_{\mu \nu}, \\
\nabla_{\mu} F^{\mu \nu} & =-\kappa D^{\nu} .
\end{aligned}
$$

We can split the fields $g_{\mu \nu}$ and $A_{\mu}$ in their zeroth and first order parts,

$$
g_{\mu \nu}=g_{\mu \nu}^{0}+g_{\mu \nu}^{1}, \quad A_{\mu}=A_{\mu}^{0}+A_{\mu}^{1},
$$

where $g_{\mu \nu}^{0}$ and $A_{\mu}^{0}$ satisfy the zeroth order equations,

$$
\Lambda g_{\mu \nu}^{0}=-G_{\mu \nu}^{0}+8 \pi T_{\mu \nu}^{0}, \quad \nabla_{\mu}^{0} F^{0 \mu \nu}=0
$$

with $\nabla_{\mu}^{0}, G_{\mu \nu}^{0}$, and $T_{\mu \nu}^{0}$ defined in terms of the zeroth order fields, and $g_{\mu \nu}^{1}, A_{\mu}^{1}$ are linear in $\kappa$.

Let us consider the spherically symmetric case, then we have the Reissner-Nordström-de Sitter solution for the lowest order equations,

$$
g_{\mu \nu}^{0}=\left(\begin{array}{cccc}
-f(r) & 0 & 0 & 0 \\
0 & f(r)^{-1} & 0 & 0 \\
0 & 0 & r^{2} & 0 \\
0 & 0 & 0 & r^{2} \sin ^{2} \theta
\end{array}\right)
$$


with $f(r)=1-\frac{2 m}{r}-\frac{\Lambda r^{2}}{3}+\frac{q^{2}}{r^{2}}$, and the corresponding

$$
F_{\mu \nu}^{0}=\left(\begin{array}{cccc}
0 & \frac{q}{r^{2}} & 0 & 0 \\
-\frac{q}{r^{2}} & 0 & 0 & 0 \\
0 & 0 & 0 & 0 \\
0 & 0 & 0 & 0
\end{array}\right)
$$

We can write the equations at the next order in $\kappa$ as

$$
\begin{gathered}
\Lambda g_{\mu \nu}^{0}+\Lambda g_{\mu \nu}^{1}=-G_{\mu \nu}^{0}-G_{\mu \nu}^{1}+8 \pi T_{\mu \nu}^{0}+8 \pi T_{\mu \nu}^{1}-\kappa C_{\mu \nu}, \\
\nabla_{\mu}^{0} F^{0 \mu \nu}+\nabla_{\mu}^{0} F^{1 \mu \nu}+\nabla_{\mu}^{1} F^{0 \mu \nu}=-\kappa D^{\nu},
\end{gathered}
$$

where $G_{\mu \nu}^{1}$ and $T_{\mu \nu}^{1}$ are the $\mathcal{O}(\kappa)$ parts of $G_{\mu \nu}$ and $T_{\mu \nu}$, and $C_{\mu \nu}$ and $D^{\nu}$ are defined as functions of the zeroth-order fields $g_{\mu \nu}^{0}$ and $F_{\mu \nu}^{0}$, and have the form

$$
\begin{gathered}
C_{\mu \nu}=\left[\left(S_{\mu \nu}-\frac{1}{4} g_{\mu \nu} S\right)-\nabla_{\alpha} F_{\nu}^{\alpha} \nabla_{\beta} F_{\mu}^{\beta}-2 F_{\mu \alpha}\left(R^{\alpha \beta}+2 F^{\alpha \gamma} F_{\gamma}^{\beta}\right) F_{\beta \nu}\right. \\
\left.-H_{\mu \nu}^{\prime}-\frac{1}{2} F^{2}\left(R_{\mu \nu}+F_{\mu \beta} F_{\nu}{ }^{\beta}\right)\right], \\
D^{\nu}=\left[\nabla_{\alpha}\left(F^{\alpha \nu}\left(\frac{1}{2} R+\frac{1}{2} F^{2}\right)\right)-4 \nabla_{\alpha}\left(F^{\alpha \beta} F_{\beta \gamma} F^{\gamma \nu}\right)-2 \nabla_{\alpha}\left(R^{\alpha \beta} F_{\beta}{ }^{\nu}\right)\right. \\
\left.-\frac{1}{2} \square\left(\nabla_{\alpha} F^{\alpha \nu}\right)+\frac{1}{2} \nabla_{\alpha} \nabla^{\nu}\left(\nabla_{\beta} F^{\beta \alpha}\right)\right] .
\end{gathered}
$$

Given $g_{\mu \nu}^{0}$ and $F_{\mu \nu}^{0}$, Eq. (5.7) reduces to finding the solution to

$$
\begin{gathered}
\Lambda g_{\mu \nu}^{1}=-G_{\mu \nu}^{1}+8 \pi T_{\mu \nu}^{1}-\kappa C_{\mu \nu}, \\
\nabla_{\mu}^{0} F^{1 \mu \nu}+\nabla_{\mu}^{1} F^{0 \mu \nu}=-\kappa D^{\nu} .
\end{gathered}
$$

We can calculate $C_{\mu \nu}$ and $D^{\nu}$ in a straightforward manner for the Reissner-Nordström-de Sitter solution,

$$
\begin{aligned}
& \kappa C_{\mu \nu}=\left(\begin{array}{cccc}
-f(r)\left(-\frac{9 \kappa q^{4}}{2 r^{8}}-\frac{\kappa \Lambda q^{2}}{r^{4}}\right) & 0 & 0 & 0 \\
0 & \left(-\frac{9 \kappa q^{4}}{2 r^{8}}-\frac{\kappa \Lambda q^{2}}{r^{4}}\right)(f(r))^{-1} & 0 & 0 \\
0 & 0 & r^{2}\left(\frac{3 \kappa q^{4}}{2 \Lambda r^{8}}+\frac{\kappa q^{2}}{r^{4}}\right) & 0 \\
0 & 0 & 0 & r^{2} \sin ^{2} \theta\left(\frac{3 \kappa q^{4}}{2 \Lambda r^{8}}+\frac{\kappa q^{2}}{r^{4}}\right)
\end{array}\right) \\
& \kappa D^{\nu}=\left(\frac{12 \kappa q^{3}}{r^{7}}, 0,0,0\right) .
\end{aligned}
$$


As might have been expected, the metric at this order remains spherically symmetric, and that the $\mathcal{O}(\kappa)$ correction for $A_{\mu}^{0}$ is only for the time component. If we now write the metric $g_{\mu \nu}$ and the four potential $A_{\mu}$

$$
\begin{aligned}
g_{\mu \nu} & =\left(\begin{array}{cccc}
-f(r)-\kappa A(r) & 0 & 0 & 0 \\
0 & (f(r)+\kappa A(r))^{-1} & 0 & 0 \\
0 & 0 & r^{2} & 0 \\
0 & 0 & 0 & r^{2} \sin ^{2} \theta
\end{array}\right), \\
A_{\mu} & =\left(\frac{q}{r}+\kappa B(r), 0,0,0\right) .
\end{aligned}
$$

Putting these in Eq. (5.10), we find that to $\mathcal{O}(\kappa)$, the following are the solutions for the metric and the electromagnetic field,

$$
\begin{aligned}
g_{\mu \nu} & =\left(\begin{array}{cccc}
-\left(f(r)+\kappa\left(\frac{3 q^{4}}{10 r^{6}}-\frac{\Lambda q^{2}}{r^{2}}\right)\right) & 0 & 0 \\
0 & \left(f(r)+\kappa\left(\frac{3 q^{4}}{10 r^{6}}-\frac{\Lambda q^{2}}{r^{2}}\right)\right)^{-1} & 0 & 0 \\
0 & 0 & r^{2} & 0 \\
0 & 0 & 0 & r^{2} \sin ^{2} \theta
\end{array}\right), \\
F_{\mu \nu} & =\left(\begin{array}{cccc}
0 & \frac{q}{r^{2}}+\frac{3 \kappa q^{3}}{r^{6}} & 0 & 0 \\
-\frac{q}{r^{2}}-\frac{3 \kappa q^{3}}{r^{6}} & 0 & 0 & 0 \\
0 & 0 & 0 & 0 \\
0 & 0 & 0 & 0
\end{array}\right) .
\end{aligned}
$$

It can also be shown that the above expressions satisfy the $\mathcal{O}(\kappa)$ trace equation Eq. 4.8, thus confirming that it is indeed the solution at this order. The trace equation for this solution works out to

$$
R=4 \Lambda+\frac{6 \kappa q^{4}}{r^{8}},
$$

which indicates that there is no singularity at finite $r$ for this solution.

It is easy to see how the iterative procedure can be extended to include higher order terms in $\kappa$. For example, at the next order in $\kappa$, we will have to expand the action of Eq. (3.11) to $\mathcal{O}\left(\kappa^{3}\right)$. This will produce equations for the $\mathcal{O}\left(\kappa^{2}\right)$ terms of the metric and the electromagnetic field tensor, which look like

$$
\begin{gathered}
\Lambda g_{\mu \nu}^{(2)}=-G_{\mu \nu}^{(2)}+8 \pi T_{\mu \nu}^{(2)}-\kappa C_{\mu \nu}^{\prime}, \\
\nabla_{\mu}^{0} F^{(2) \mu \nu}+\nabla_{\mu}^{1} F^{1 \mu \nu}+\nabla_{\mu}^{(2)} F^{0 \mu \nu}=-\kappa D^{\prime \nu},
\end{gathered}
$$


where now $G_{\mu \nu}^{(2)}, T_{\mu \nu}^{(2)}$ and $F^{(2) \mu \nu}$ are the $\mathcal{O}\left(\kappa^{2}\right)$ terms in $G_{\mu \nu}, T_{\mu \nu}$, and $F^{\mu \nu}$, and $C_{\mu \nu}^{\prime}$ and $D^{\prime \nu}$ are $\mathcal{O}(\kappa)$ functions analogous to Eq. (5.8) and Eq. (5.9), but including additional terms coming from the higher order expansion of the action, and calculated using the $\mathcal{O}(\kappa)$ field solutions. Extending in this manner, we can find a solution to the theory to any order in $\kappa$.

We started out with the goal of finding a natural way of adding matter fields to the Eddington-inspired Born-Infeld action of gravity. The Kaluza procedure is certainly natural in the sense that it is geometric, but it deviates from the philosophy of the Eddington action in that it has to be written purely as a metric theory, otherwise it would not be possible to interpret $g_{\mu 5}$ as components of the electromagnetic potential.

Since the five-dimensional action comes from the expansion of the square root of a polynomial, it is non-polynomial in nature, and thus the coupling between electromagnetism and gravity is highly nonlinear. However, we can expand in powers of curvature (equivalently in powers of $\kappa$ ) and find and solve the equations of motion term by term. Applying this iterative procedure to the lowest order electric Reissner-Nordström-de Sitter black hole solution, we found the $\mathcal{O}(\kappa)$ correction. At least at this order, there is no singularity at finite radius, unlike the 'surface singularities' which plague four-dimensional Eddington-inspired Born-Infeld gravity with minimally added matter.

\section{Acknowledgments}

We thank the anonymous referee, whose critical comments helped us make significant additions to the paper. 


\section{Appendix A: Non-trivial $\Phi$}

If we do not set $\Phi=1$ in the five-dimensional metric Eq. (3.1), we will find the following Ricci tensor components,

$$
\begin{aligned}
\hat{R}_{\mu \nu}= & R_{\mu \nu}-\Phi^{-1} \nabla_{\mu} \nabla_{\nu} \Phi+\frac{\alpha^{4}}{4} F^{\beta \gamma} F_{\beta \gamma} \Phi^{4} A_{\mu} A_{\nu}-\frac{\alpha^{2}}{2}\left[3 \Phi \partial_{\beta} \Phi\left(F_{\mu}^{\beta} A_{\nu}+F_{\nu}^{\beta} A_{\mu}\right)\right. \\
& \left.+2 A_{\mu} A_{\nu} \Phi \square \Phi+\Phi^{2}\left(A_{\mu} \nabla_{\beta} F_{\nu}^{\beta}+A_{\nu} \nabla_{\beta} F_{\mu}^{\beta}+F_{\beta \mu} F_{\nu}^{\beta}\right)\right] \\
\hat{R}_{\mu 5}= & \frac{\alpha^{3}}{4} F^{\beta \gamma} F_{\beta \gamma} \Phi^{4} A_{\mu}-\frac{\alpha}{2}\left(3 \Phi \partial^{\beta} \Phi F_{\beta \mu}+\Phi^{2} \nabla_{\beta} F_{\mu}^{\beta}+2 \Phi \square \Phi A_{\mu}\right) \\
\hat{R}_{55}= & \frac{\alpha^{2}}{4} \Phi^{4} F^{\beta \gamma} F_{\beta \gamma}-\Phi \square \Phi .
\end{aligned}
$$

The five-dimensional Ricci scalar is calculated from this to be

$$
\hat{R}=R-2 \Phi^{-1} \square \Phi-\frac{\alpha^{2}}{4} \Phi^{2} F^{\beta \gamma} F_{\beta \gamma}
$$

[1] M. Born and L. Infeld, Proc. Roy. Soc. Lond. A 144, 425 (1934).

[2] J. A. Feigenbaum, P. G. O. Freund and M. Pigli, Phys. Rev. D 57, 4738 (1998)

[3] S. Deser and G. W. Gibbons, Class. Quant. Grav. 15, L35 (1998)

[4] J. A. Feigenbaum, Phys. Rev. D 58, 124023 (1998)

[5] D. Comelli and A. Dolgov, JHEP 0411, 062 (2004)

[6] M. Banados and P. G. Ferreira, Phys. Rev. Lett. 105, 011101 (2010)

[7] J. H. C. Scargill, M. Banados and P. G. Ferreira, Phys. Rev. D 86, 103533 (2012)

[8] M. N. R. Wohlfarth, Class. Quant. Grav. 21, 1927 (2004) [Erratum-ibid. 21, 5297 (2004)]

[9] T. Delsate and J. Steinhoff, Phys. Rev. Lett. 109, 021101 (2012)

[10] T. Delsate and J. Steinhoff, arXiv:1301.1233 [gr-qc]

[11] P. Pani and T. P. Sotiriou, Phys. Rev. Lett. 109, 251102 (2012)

[12] G. W. Gibbons and D. A. Rasheed, Nucl. Phys. B 454, 185 (1995)

[13] S. Deser, J. G. McCarthy and O. Sarioglu, Class. Quant. Grav. 16, 841 (1999)

[14] I. Bialynicki-Birula, In Jancewicz, B. ( Ed.), Lukierski, J. ( Ed.): Quantum Theory Of Particles and Fields, World Sci. 1984, p.31.

[15] J. Plebanski, Lectures On Non Linear Electrodynamics, NORDITA, 1970.

[16] S. Deser and O. Sarioglu, Phys. Lett. B 423, 369 (1998) 
[17] A. S. Eddington, The Mathematical theory of Relativity, Cambridge Univ. Pr., Cambridge, UK (1924).

[18] E. Schrödinger, Space-time structure, Cambridge Univ. Pr., Cambridge, UK (1954).

[19] M. Banados, P. G. Ferreira and C. Skordis, Phys. Rev. D 79, 063511 (2009)

[20] D. N. Vollick, Phys. Rev. D 69, 064030 (2004)

[21] D. N. Vollick, Phys. Rev. D 72, 084026 (2005)

[22] R. Wald General Relativity, The University of Chicago Pr., Chicago, U.S.A. (1984).

[23] T. Kaluza, Sitzungsber. Preuss. Akad. Wiss. Berlin (Math. Phys. ) 1921, 966 (1921).

[24] P. Jordan, Ann. Phys. (Leipzig) 219, 1 (1947). 